ESJ Social Sciences

\title{
Análisis de la Actitud Emprendedora de los Estudiantes Varones del Tecnológico Nacional de México Campus Tepeaca
}

\author{
María Luisa Juárez Hernández, PhD \\ Manuel González Pérez, PhD \\ Rafael Tovany-León, M.C \\ Ing. José Atonaltzin Maldonado-Ortiz
}

Tecnológico Nacional de México campus Tepeaca, Puebla, México

\section{Doi:10.19044/esj.2021.v17n32p112}

Submitted: 20 July 2021

Accepted: 07 September 2021

Published: 30 September 2021
Copyright 2021 Author(s)

Under Creative Commons BY-NC-ND

4.0 OPEN ACCESS

Cite As:

Juárez Hernández M.L., González Pérez M., Tovany-León R. \& Maldonado-Ortiz J.A. (2021). Análisis de la Actitud Emprendedora de los Estudiantes Varones del Tecnológico Nacional de México Campus Tepeaca. European Scientific Journal, ESJ, 17 (32), 112.

https://doi.org/10.19044/esj.2021.v17n32p112

\section{Resumen}

El espíritu juvenil de los estudiantes de convertirse en emprendedores es notorio a simple vista. Sin embargo, el objetivo general de esta investigación fue analizar la actitud emprendedora de los estudiantes varones del Tecnológico Nacional de México campus Tepeaca. Se encuestó a ciento setenta y dos estudiantes varones. El cuestionario fue diseñado y validado por expertos en el área. La validación se llevó a cabo por un método nuevo; éste consiste en aplicar un análisis factorial de Pearson para las correlaciones lineales y chi cuadrado para las asociaciones no lineales. Se le aplican cinco postulados para depurar y validar cada ítem. Los ítems se toman como factores o variables independientes para contrastar las hipótesis de trabajo. El muestreo fue aleatorio. La matriz de resultados obtenida después de aplicar la correlación de Pearson arrojó tres correlaciones por encima de 0.7. Con estas correlaciones se validaron seis hipótesis lineales. Solo se seleccionaron ocho hipótesis no lineales validadas por chi cuadrado debido al espacio limitado de un artículo científico. En general, se diseñaron 400 hipótesis de trabajo en el orden de 20 ítems tomados en orden de dos a dos. Se concluye que los estudiantes encuestados poseen amplio conocimiento del emprendimiento. Éstos están preparados para la innovación, creación y éxito en las empresas 
tanto de su propiedad como las empresas donde presten sus servicios.

Palabras clave: Espíritu emprendedor, Estudiantes, Tecnológico nacional de México, Tepeaca Puebla

\title{
Analysis of the Entrepreneurial Attitude of Male Students of the Tecnológico Nacional de México Campus Tepeaca
}

\author{
María Luisa Juárez Hernández, PhD \\ Manuel González Pérez, PhD \\ Rafael Tovany-León, M.C \\ Ing. José Atonaltzin Maldonado-Ortiz
}

Tecnológico Nacional de México campus Tepeaca, Puebla, México

\begin{abstract}
The youthful spirit of students to become entrepreneurs is apparent to the naked eye. However, the general objective of this research was to analyze the entrepreneurial attitude of male students from the Tecnológico Nacional de México campus, Tepeaca. One hundred and seventy-two male students were surveyed. The questionnaire was designed and validated by experts in the area. The validation was carried out by a new method; This consists of applying a Pearson factorial analysis for linear correlations and chi-square for nonlinear associations. Five postulates are applied to debug and validate each item. The items are taken as factors or independent variables to contrast the working hypotheses. The sampling was random. The results matrix obtained after applying Pearson's correlation yielded three correlations above 0.7 . With these correlations, six linear hypotheses were validated. Only eight chi-squarevalidated nonlinear hypotheses were selected due to the limited space of a scientific article. In general, 400 working hypotheses were designed in the order of 20 items taken in two to two. It is concluded that the surveyed students have extensive knowledge of entrepreneurship. They are prepared for innovation, creation, and success in the companies they own and the companies where they provide their services.
\end{abstract}

Keywords: Entrepreneurial, Attitude, Students, National Technological Institute of Mexico, Tepeaca Puebla 


\section{Introduction}

Para Neira et al. (2013) el deseo de los estudiantes para convertirse en empresarios bajo una actitud emprendedora está influenciado por el desarrollo de competencias relacionadas con la gestión y por la percepción de las normas sociales sobre el espíritu emprendedor. Ruiz et al. (2014) hacen evidente que en el caso de los hombres la autoeficacia es la variable principal de la actitud emprendedora y que influye en la puesta en marcha de iniciativas empresariales, y en el caso de las mujeres es la controlabilidad percibida. De Jorge (2013), dice que los factores que influyen en la actitud emprendedora de los hombres están relacionados con las características de edad, es decir, a mayor edad mayor intención emprendedora, y también a la existencia de algún familiar empresario.

Espíritu y Moreno (2011) infieren que los estudiantes tienen una estrecha relación de la necesidad de logro, control interno y propensión al riesgo como factores de personalidad que potencian su intención de crear su propia empresa. Además, Feliz J. (2017) indica que los factores sociodemográficos no son determinantes en el desarrollo de una actitud emprendedora en los estudiantes, en cambio, factores psicológicos y sociológicos como el capital social, las redes sociales y el entorno familiar influyen de manera preponderante en su comportamiento emprendedor.

Por otro lado, Borrayo et al. (2019) han observado que la actitud emprendedora de los estudiantes universitarios va disminuyendo durante su estancia en la universidad debido en menor medida a factores como el miedo al fracaso, condiciones económicas y falta de asesoría profesional y en mayor medida a falta de programas de formación de emprendedores y de negocios. Ordoñez (2017) ha identificado también que los factores que influyen en las habilidades emprendedoras de los universitarios son la capacidad creativa, el compromiso y la responsabilidad. Adicionalmente, condicionantes como una mayor edad del estudiante y antecedentes de emprendimiento en su familia presentan una mayor influencia en su habilidad emprendedora. Hernández (2019) determinó en su estudio en estudiantes universitarios que los rasgos de personalidad de extroversión, y apertura a experiencias tienen una relación estrecha con la aptitud para emprender, contrario al neuroticismo, amabilidad y responsabilidad y en menor medida a diferencias entre hombres y mujeres.

Méndez et al. (2017), observaron que, en los estudiantes universitarios al avanzar su retícula académica, va disminuyendo su interés en el desarrollo del emprendimiento, si éste no es influenciado por factores que detonen estas intenciones como el estudiar, analizar y proponer temas y proyectos de emprendimiento a lo largo de toda su carrera y únicamente con cursos aislados. Espíritu emprendedor.

Para Cruz, Barahona \& Escudero (2005) es necesario desarrollar estrategias para que los estudiantes universitarios crezcan su espíritu 
emprendedor como formación adicional a la de sus competencias universitarias, teniendo un enfoque prioritario en la generación de experiencias laborales previas a su egreso, así como a la de la formación complementaria en emprendimiento. Rincón y Álvarez (2020) mencionan que existen diferencias en la media de percepción entre grupos de géneros (mujeres y hombres) estudiantes universitarios para el desarrollo del espíritu emprendedor considerando como parámetros las variables de información, asesoría, competencias y ambiente universitarios que son impulsoras del espíritu emprendedor universitario.

De acuerdo con el contexto anterior se enuncia el objetivo general de esta investigación que fue analizar la actitud emprendedor de los estudiantes varones del tecnológico nacional de México campus Tepeaca.

\section{Metodología:}

Se encuestaron 172 estudiantes varones del TecNM campus Tepeaca México. El cuestionario fue diseñado y validado por expertos en el área. Se diseñó con escala de Likert para validación lineal y luego se dicotomizó para validar las hipótesis no lineales. El muestreo fue totalmente aleatorio.

La validación del cuestionario se hizo aplicando una correlación múltiple de los ítems. La investigación fue cualitativa y paramétrica.

La selección de las hipótesis validadas que se publican en este artículo fue hecha por expertos, se omitieron algunas por razones de espacio, es decir se publican las hipótesis más significativas para el tema del artículo.

El diseño del cuestionario derivó de una matriz de operacionalización tal y como se muestra en la tabla 1.

La tabla 1 tiene tres columnas. En la primera columna se presentan las variables, en la segunda columna se ubican los indicadores y en la tercera columna se redactan los ítems. Estas preguntas se seleccionaron por expertos de una lluvia de ideas, según el método de Brian Tracy.

Tabla 1. Matriz de operacionalización

\begin{tabular}{|c|c|c|}
\hline Variables & Indicador & Ítems \\
\hline $\begin{array}{c}\text { Actitud } \\
\text { emprendedora }\end{array}$ & $\begin{array}{l}\text { Actitud positiva. } \\
\text { Actitud neutra. } \\
\text { Actitud negativa. }\end{array}$ & $\begin{array}{l}\text { 1. ¿Con qué frecuencia tomas una actitud neutral } \\
\text { ante un nuevo reto de la vida? } \\
\text { 2. ¿Con qué frecuencia tomas una actitud positiva } \\
\text { ante un nuevo reto de la vida? }\end{array}$ \\
\hline $\begin{array}{c}\text { Aptitud } \\
\text { emprendedora }\end{array}$ & $\begin{array}{l}\text { Experiencia en el } \\
\text { carácter } \\
\text { emprendedor }\end{array}$ & $\begin{array}{l}\text { 3. ¿Con qué frecuencia te sientes capacitado para } \\
\text { afrontar un nuevo desafío en la escuela o el } \\
\text { trabajo? } \\
\text { 4. ¿Con qué frecuencia sientes el deseo de } \\
\text { capacitarte cuando no sabes desempeñar un buen } \\
\text { papel en la escuela o el trabajo? }\end{array}$ \\
\hline $\begin{array}{c}\text { Espíritu } \\
\text { emprendedor }\end{array}$ & $\begin{array}{c}\text { Metas claras } \\
\text { Visión } \\
\text { Inversión } \\
\end{array}$ & $\begin{array}{l}\text { 5. ¿Qué tan fuerte es tu deseo por lograr tus metas? } \\
\text { ¿Qué tan frecuente mantienes tu visión de las } \\
\text { metas a lograr? }\end{array}$ \\
\hline
\end{tabular}




\begin{tabular}{|c|c|c|}
\hline & $\begin{array}{c}\text { Paciencia } \\
\text { Enfoque } \\
\text { Capacidad de } \\
\text { adaptación } \\
\text { Perseverancia } \\
\text { Resiliencia } \\
\text { Pasión }\end{array}$ & $\begin{array}{l}\text { 7. ¿Qué tanta fe tienes para invertir o conseguir } \\
\text { inversión para lograr tu meta emprendedora? } \\
\text { 8. ¿Con qué frecuencia te echas porras en el camino } \\
\text { hacia lograr tu meta emprendedora? } \\
\text { 9. ¿Con qué frecuencia te enfocas en tu meta } \\
\text { emprendedora? } \\
\text { 10. Cuándo tienes un cambio inesperado en el camino } \\
\text { hacia tu meta emprendedora ¿Qué tan rápido te } \\
\text { adaptas a las nuevas normas o características? } \\
\text { 11. ¿Qué tan perseverante eres en el camino hacia tu } \\
\text { meta? } \\
\text { 12. ¿Qué tanto aceptas y superas los reveses que se } \\
\text { presentan en el camino a tu meta? } \\
\text { 13. ¿Con qué pasión llevas el camino rumbo a tu } \\
\text { meta? }\end{array}$ \\
\hline $\begin{array}{l}\text { Liderazgo de un } \\
\text { emprendedor. }\end{array}$ & $\begin{array}{c}\text { Liderazgo } \\
\text { Proactividad } \\
\text { Protagonismo } \\
\text { Compromiso }\end{array}$ & $\begin{array}{l}\text { 14. ¿Qué tan a menudo quitas obstáculos a los } \\
\text { integrantes de tu equipo emprendedor para su } \\
\text { crecimiento? } \\
\text { 15. ¿Con qué frecuencia resuelves conflictos entre los } \\
\text { integrantes de tu grupo? } \\
\text { 16. ¿Qué tan proactivo eres en tu equipo? } \\
\text { 17. ¿Qué tanto te involucras en los nuevos retos que } \\
\text { se presentan en tu equipo de trabajo? } \\
\text { 18. ¿Qué tan comprometido(a) te sientes con tu } \\
\text { equipo de trabajo, con tu institución o con tu } \\
\text { empresa? } \\
\text { 19. ¿Qué tanto éxito has tenido en tu equipo o equipos } \\
\text { de trabajo? } \\
\text { 20. ¿Qué tan a menudo quitas obstáculos a los } \\
\text { integrantes de tu equipo emprendedor para su } \\
\text { crecimiento? }\end{array}$ \\
\hline
\end{tabular}

La validación del cuestionario se llevó a cabo usando la metodología del Dr. Manuel González Pérez ya publicada en otros artículos (García-Arroyo et al. 2020). Esta metodología consiste en aplicar una prueba piloto. A los datos crudos se le aplican dos modelos estadísticos multifactorial. A) Correlación de Pearson para las relaciones lineales. B) Chi cuadrada para las asociaciones no lineales.

La validación por expertos se hizo aplicando cinco postulados que se muestran a continuación:

Postulado 1. Los ítems están repetidos. Falso. No hubo error de copiar y pegar.

Postulado 2. Los ítems están parafraseados. Falso. No se encontró ítem parafraseados. No hubo error por parafraseado de ideas.

Postulado 3. Los ítems están repetidos o parafraseados a propósito. Falso. Este postulado se usa para detectar una contradicción de los sujetos encuestados. 
Postulado 4. Los ítems están correlacionados por casualidad. Falso. Los expertos no detectaron este fenómeno, una correlación por casualidad.

Postulado 5. Los ítems se correlacionaron por su propia naturaleza. Verdadero. Si hay correlación, se verificó que las hipótesis dicotómicas que se formen tengan un sentido lógico o común congruente y pertinente a los objetivos.

\section{Resultados y discusión:}

La tabla 2, muestra una correlación múltiple de Pearson para validación del cuestionario. Como se puede ver, se contrastaron todos los ítems. Cada ítem se tomó como una variable independiente. De esta forma se hizo un análisis multifactorial para contraste lineal.

Tabla 2. Correlación múltiple de Pearson.

Emprendimiento hombres. TecnM campus Tepeaca.

\begin{tabular}{|c|c|c|c|c|c|c|c|c|c|c|c|c|c|c|c|c|c|c|c|c|}
\hline & Item1 & Item2 & Item3 & Item4 & Item5 & Item6 & Item7 & Item8 & Item9 & Item10 & Item11 & Item12 & Item13 & Item14 & Item15 & Item16 & Item17 & Item18 & Item19 & Item2 \\
\hline Item1 & 1 & & & & & & & & & & & & & & & & & & & \\
\hline Item2 & 0.3826 & 1 & & & & & & & & & & & & & & & & & & \\
\hline Item3 & 0.4008 & 0.605 & 1 & & & & & & & & & & & & & & & & & \\
\hline Item4 & 0.2222 & 0.473 & 0.499 & 1 & & & & & & & & & & & & & & & & \\
\hline Item5 & 0.3768 & 0.5326 & 0.4792 & 0.5213 & 1 & & & & & & & & & & & & & & & \\
\hline Item6 & 0.3325 & 0.5515 & 0.5539 & 0.4543 & 0.7476 & 1 & & & & & & & & & & & & & & \\
\hline Item7 & 0.4313 & 0.547 & 0.5016 & 0.3228 & 0.4174 & 0.5344 & 1 & & & & & & & & & & & & & \\
\hline Item8 & 0.4041 & 0.5631 & 0.4556 & 0.3351 & 0.4258 & 0.4631 & 0.5393 & 1 & & & & & & & & & & & & \\
\hline Item9 & 0.3955 & 0.5235 & 0.4768 & 0.3774 & 0.46 & 0.4963 & 0.5822 & 0.6805 & 1 & & & & & & & & & & & \\
\hline Item10 & 0.3436 & 0.4949 & 0.5023 & 0.2919 & 0.3252 & 0.4858 & 0.4951 & 0.537 & 0.5622 & 1 & & & & & & & & & & \\
\hline Item11 & 0.4019 & 0.4867 & 0.5057 & 0.3953 & 0.543 & 0.5738 & 0.4621 & 0.6139 & 0.6104 & 0.5741 & 1 & & & & & & & & & \\
\hline Item12 & 0.4405 & 0.5018 & 0.494 & 0.4232 & 0.4512 & 0.4996 & 0.5194 & 0.5143 & 0.6177 & 0.6092 & 0.6561 & 1 & & & & & & & & \\
\hline Item13 & 0.4537 & 0.5637 & 0.5564 & 0.4369 & 0.5927 & 0.6292 & 0.5642 & 0.5772 & 0.6916 & 0.5214 & 0.7351 & 0.6721 & 1 & & & & & & & \\
\hline Item14 & 0.2941 & 0.399 & 0.453 & 0.2482 & 0.3429 & 0.4271 & 0.3841 & 0.398 & 0.4443 & 0.4978 & 0.5061 & 0.4605 & 0.436 & 1 & & & & & & \\
\hline Item15 & 0.3455 & 0.3249 & 0.3727 & 0.2044 & 0.1749 & 0.2748 & 0.3167 & 0.2913 & 0.3756 & 0.3839 & 0.3175 & 0.4203 & 0.3382 & 0.5395 & 1 & & & & & \\
\hline Item16 & 0.2599 & 0.3662 & 0.4148 & 0.3704 & 0.3283 & 0.4559 & 0.3101 & 0.4508 & 0.4682 & 0.4955 & 0.4319 & 0.3962 & 0.4543 & 0.4749 & 0.4456 & 1 & & & & \\
\hline Item17 & 0.375 & 0.4422 & 0.5379 & 0.3805 & 0.3336 & 0.4687 & 0.471 & 0.512 & 0.5047 & 0.5758 & 0.5858 & 0.511 & 0.5572 & 0.5111 & 0.5363 & 0.668 & 1 & & & \\
\hline Item18 & 0.2712 & 0.3626 & 0.4964 & 0.412 & 0.4242 & 0.4689 & 0.3426 & 0.4854 & 0.4645 & 0.4622 & 0.532 & 0.4757 & 0.4217 & 0.3928 & 0.3868 & 0.5098 & 0.6317 & 1 & & \\
\hline Item19 & 0.3292 & 0.3886 & 0.5075 & 0.4248 & 0.3592 & 0.4164 & 0.4336 & 0.4869 & 0.4978 & 0.4177 & 0.4741 & 0.4373 & 0.4321 & 0.3975 & 0.4071 & 0.4839 & 0.5749 & 0.5982 & 1 & \\
\hline Item 20 & 0.3269 & 0.3175 & 0.4254 & 0.2606 & 0.296 & 0.4006 & 0.2987 & 0.3384 & 0.3848 & 0.3595 & 0.3834 & 0.3769 & 0.4017 & 0.5557 & 0.504 & 0.4571 & 0.5393 & 0.4675 & 0.485 & 1 \\
\hline
\end{tabular}

Se usó la plantilla Excel para hacer el análisis general de la matriz de datos crudos. Esta matriz se generó con los resultados obtenidos después de aplicar el cuestionario a 172 estudiantes varones del tecnológico de Tepeaca. Se puede observar que solo se obtuvieron tres correlaciones arriba de 0.69 (correlación muy alta).

En la tabla 3, columna 1, se presentan los pares ordenados con el número de cada ítem del cuestionario. En las columnas 2 y 3 se observan los ítems que se van a cruzar para formar las inferencias. En la columna 4 se exhiben las inferencias de los ítems cruzados. En la columna 5 se presentan los coeficientes de correlación de Pearson arriba de 0.69. Este coeficiente permite tener una correlación lineal fuerte. Por último, en la columna 6 se presentan las hipótesis comprobadas o tesis. Se observa una buena secuencia lógica de las inferencias para generar las hipótesis de trabajo comprobadas. Estas hipótesis concuerdan con la matriz de operacionalización y son derivadas de las hipótesis de investigación.

Las hipótesis de investigación son muy generales se hizo notar que era necesario una subclasificación de éstas. 
September 2021 edition Vol.17, No.32

Tabla 3. Tabla de hipótesis comprobadas por medio de la correlación múltiple de Pearson

\begin{tabular}{|c|c|c|c|c|c|}
\hline $\begin{array}{l}\text { Par } \\
\text { ordenado }\end{array}$ & ItemA & ÍtemB & Inferencia & $\begin{array}{l}\text { Coeficiente de } \\
\text { correlación de } \\
\text { Pearson }\end{array}$ & $\begin{array}{l}\text { Hipótesis } \\
\text { comprobada }\end{array}$ \\
\hline$(5,6)$ & $\begin{array}{l}\text { ¿Qué tan fuerte } \\
\text { es tu deseo por } \\
\text { lograr tus } \\
\text { metas? }\end{array}$ & $\begin{array}{l}\text { ¿Qué tan } \\
\text { frecuente } \\
\text { mantienes tu } \\
\text { visión de las } \\
\text { metas a lograr? }\end{array}$ & $\begin{array}{l}\text { Si tienes un } \\
\text { fuerte deseo por } \\
\text { lograr tus metas } \\
\text { entonces } \\
\text { mantienes tu } \\
\text { visión para } \\
\text { lograrlas. }\end{array}$ & 0.7476 & $\begin{array}{l}\text { El fuerte deseo } \\
\text { por lograr las } \\
\text { metas } \\
\text { mantiene la } \\
\text { visión para } \\
\text { lograrlas. }\end{array}$ \\
\hline$(6,5)$ & $\begin{array}{l}\text { ¿Qué tan } \\
\text { frecuente } \\
\text { mantienes tu } \\
\text { visión de las } \\
\text { metas a lograr? }\end{array}$ & $\begin{array}{l}\text { ¿Qué tan fuerte } \\
\text { es tu deseo } \\
\text { ardiente por } \\
\text { lograr tus } \\
\text { metas? }\end{array}$ & $\begin{array}{l}\text { Si mantienes tu } \\
\text { visión por lograr } \\
\text { tus metas, } \\
\text { entonces sientes } \\
\text { un deseo ardiente } \\
\text { por lograrlas. }\end{array}$ & 0.7476 & $\begin{array}{l}\text { Mantener la } \\
\text { visión por } \\
\text { lograr las } \\
\text { metas. sostiene } \\
\text { un fuerte deseo } \\
\text { por lograrlas. }\end{array}$ \\
\hline$(9,13)$ & $\begin{array}{l}\text { ¿Con qué } \\
\text { frecuencia te } \\
\text { enfocas en tu } \\
\text { meta } \\
\text { emprendedora? }\end{array}$ & $\begin{array}{l}\text { ¿Con qué } \\
\text { pasión llevas } \\
\text { el camino } \\
\text { rumbo a tu } \\
\text { meta? }\end{array}$ & $\begin{array}{l}\mathrm{Si} \\
\text { frecuentemente } \\
\text { enfocas tu meta } \\
\text { emprendedora, } \\
\text { entonces llevas } \\
\text { con pasión el } \\
\text { camino rubo a } \\
\text { ella. }\end{array}$ & 0.691588166 & $\begin{array}{l}\text { El enfoque } \\
\text { frecuente en la } \\
\text { meta } \\
\text { emprendedora } \\
\text { genera pasión } \\
\text { en el camino } \\
\text { rumbo a ella. }\end{array}$ \\
\hline$(13,9)$ & $\begin{array}{l}\text { ¿Con qué } \\
\text { pasión llevas } \\
\text { el camino } \\
\text { rumbo a tu } \\
\text { meta? }\end{array}$ & $\begin{array}{l}\text { ¿Con qué } \\
\text { frecuencia te } \\
\text { enfocas en tu } \\
\text { meta } \\
\text { emprendedora? }\end{array}$ & $\begin{array}{l}\text { Si con frecuencia } \\
\text { sientes pasión en } \\
\text { el camino rumbo } \\
\text { a la meta, } \\
\text { entonces } \\
\text { mantienes el } \\
\text { enfoque en la } \\
\text { meta } \\
\text { emprendedora. }\end{array}$ & 0.691588166 & $\begin{array}{l}\text { La pasión en el } \\
\text { camino rumbo } \\
\text { a la meta } \\
\text { emprendedora } \\
\text { genera } \\
\text { enfoque } \\
\text { constante } \\
\text { hacia ella. }\end{array}$ \\
\hline$(11,13)$ & $\begin{array}{l}\text { ¿Qué tan } \\
\text { perseverante } \\
\text { eres en el } \\
\text { camino hacia } \\
\text { tu meta? }\end{array}$ & $\begin{array}{l}\text { ¿Con qué } \\
\text { pasión llevas } \\
\text { el camino } \\
\text { rumbo a tu } \\
\text { meta? }\end{array}$ & $\begin{array}{l}\text { Si eres } \\
\text { perseverante en } \\
\text { el camino hacia } \\
\text { tu meta, entonces } \\
\text { llevas con pasión } \\
\text { el camino hacia } \\
\text { ella. }\end{array}$ & 0.735131504 & $\begin{array}{l}\text { La } \\
\text { perseverancia } \\
\text { en el camino } \\
\text { hacia la meta } \\
\text { genera pasión. }\end{array}$ \\
\hline$(13,11)$ & $\begin{array}{l}\text { ¿Con qué } \\
\text { pasión llevas } \\
\text { el camino } \\
\text { rumbo a tu } \\
\text { meta? }\end{array}$ & $\begin{array}{l}\text { ¿Qué tan } \\
\text { perseverante } \\
\text { eres en el } \\
\text { camino hacia } \\
\text { tu meta? }\end{array}$ & $\begin{array}{l}\text { Si llevas con } \\
\text { pasión el camino } \\
\text { rumbo a la meta, } \\
\text { generas } \\
\text { perseverancia en } \\
\text { el mismo }\end{array}$ & 0.735131504 & $\begin{array}{l}\text { La pasión } \\
\text { rumbo a la } \\
\text { meta genera } \\
\text { perseverancia } \\
\text { en el mismo } \\
\text { sentido. }\end{array}$ \\
\hline
\end{tabular}


Tabla 4. Algunas hipótesis comprobadas por medio de chi cuadrado dicotómica con un grado de libertad... 3.84 como punto de inflexión del chi cuadrado

\begin{tabular}{|c|c|c|c|c|c|}
\hline $\begin{array}{l}\text { Par } \\
\text { ordenado }\end{array}$ & ItemA & ÍtemB & Inferencia & $\begin{array}{l}\text { Chi } \\
\text { cuadrado }\end{array}$ & $\begin{array}{l}\text { Hipótesis } \\
\text { comprobada }\end{array}$ \\
\hline$(2,3)$ & $\begin{array}{l}\text { ¿Con qué } \\
\text { frecuencia } \\
\text { tomas una } \\
\text { actitud positiva } \\
\text { ante un nuevo } \\
\text { reto de la vida? }\end{array}$ & $\begin{array}{l}\text { ¿Con qué } \\
\text { frecuencia te } \\
\text { sientes } \\
\text { capacitado para } \\
\text { afrontar un } \\
\text { nuevo desafío } \\
\text { en la escuela o } \\
\text { el trabajo? }\end{array}$ & $\begin{array}{l}\text { Si tomas una actitud } \\
\text { positiva ante un } \\
\text { nuevo reto de la } \\
\text { vida entonces te } \\
\text { sientes capacitado } \\
\text { para afrontar un } \\
\text { nuevo desafío en la } \\
\text { escuela o el trabajo }\end{array}$ & 39.21126133 & $\begin{array}{l}\text { La actitud } \\
\text { positiva ante un } \\
\text { nuevo reto de la } \\
\text { vida genera } \\
\text { capacidad para } \\
\text { afrontar un } \\
\text { nuevo desafío en } \\
\text { la escuela o el } \\
\text { trabajo }\end{array}$ \\
\hline$(2,4)$ & $\begin{array}{l}\text { ¿Con qué } \\
\text { frecuencia } \\
\text { tomas una } \\
\text { actitud positiva } \\
\text { ante un nuevo } \\
\text { reto de la vida? }\end{array}$ & $\begin{array}{l}\text { ¿Con qué } \\
\text { frecuencia } \\
\text { sientes el deseo } \\
\text { de capacitarte } \\
\text { cuando no sabes } \\
\text { desempeñar un } \\
\text { buen papel en la } \\
\text { escuela o el } \\
\text { trabajo? }\end{array}$ & $\begin{array}{l}\text { Si timas una actitud } \\
\text { positiva ante un } \\
\text { nuevo reto de la } \\
\text { vida, entonces } \\
\text { sientes el deseo de } \\
\text { capacitarte cuando } \\
\text { no sabes } \\
\text { desempenar un buen } \\
\text { papel en el trabajo o } \\
\text { la escuela. }\end{array}$ & 10.8472866 & $\begin{array}{l}\text { La actitud } \\
\text { positiva ante un } \\
\text { nuevo reto de la } \\
\text { vida genera el } \\
\text { deseo de } \\
\text { capacitarse } \\
\text { cuando no se } \\
\text { sabe } \\
\text { desempeñar un } \\
\text { buen papel en la } \\
\text { escuela o el } \\
\text { trabajo. }\end{array}$ \\
\hline$(2,7)$ & $\begin{array}{l}\text { ¿Con qué } \\
\text { frecuencia } \\
\text { tomas una } \\
\text { actitud positiva } \\
\text { ante un nuevo } \\
\text { reto de la vida? }\end{array}$ & $\begin{array}{l}\text { ¿Qué tanta fe } \\
\text { tienes para } \\
\text { invertir o } \\
\text { conseguir } \\
\text { inversión para } \\
\text { lograr tu meta } \\
\text { emprendedora? }\end{array}$ & $\begin{array}{l}\text { Si tomas una actitud } \\
\text { positiva ante un } \\
\text { nuevo reto de la } \\
\text { vida entonces tienes } \\
\text { fe para invertir o } \\
\text { conseguir inversión } \\
\text { para lograr tu meta } \\
\text { emprendedora }\end{array}$ & 39.21126133 & $\begin{array}{l}\text { La actitud } \\
\text { positiva ante un } \\
\text { nuevo reto de la } \\
\text { vida mantiene la } \\
\text { fe para invertir o } \\
\text { conseguir } \\
\text { inversión para } \\
\text { lograr la meta. }\end{array}$ \\
\hline$(3,7)$ & $\begin{array}{l}\text { ¿Con qué } \\
\text { frecuencia te } \\
\text { sientes } \\
\text { capacitado } \\
\text { para afrontar } \\
\text { un nuevo } \\
\text { desafío en la } \\
\text { escuela o el } \\
\text { trabajo? }\end{array}$ & $\begin{array}{l}\text { ¿Qué tanta fe } \\
\text { tienes para } \\
\text { invertir o } \\
\text { conseguir } \\
\text { inversión para } \\
\text { lograr tu meta } \\
\text { emprendedora? }\end{array}$ & $\begin{array}{l}\text { Si te sientes } \\
\text { capacitado para } \\
\text { afrontar un nuevo } \\
\text { desafío en la } \\
\text { escuela o el trabajo } \\
\text { entonces tienes fe } \\
\text { para invertir o } \\
\text { conseguir inversión } \\
\text { para lograr tu meta } \\
\text { emprendedora }\end{array}$ & 35.09027844 & $\begin{array}{l}\text { La capacidad } \\
\text { para afrontar un } \\
\text { nuevo desafío en } \\
\text { la escuela o el } \\
\text { trabajo mantiene } \\
\text { la fe en invertir } \\
\text { o conseguir } \\
\text { inversión para } \\
\text { lograr la meta. }\end{array}$ \\
\hline$(5,20)$ & $\begin{array}{l}\text { ¿Qué tan fuerte } \\
\text { es tu deseo por } \\
\text { lograr tus } \\
\text { metas? }\end{array}$ & $\begin{array}{l}\text { ¿Qué tan a } \\
\text { menudo quitas } \\
\text { obstáculos a los } \\
\text { integrantes de tu } \\
\text { equipo } \\
\text { emprendedor }\end{array}$ & $\begin{array}{l}\text { Si eres fuerte es tu } \\
\text { deseo por lograr tus } \\
\text { metas entonces a } \\
\text { menudo quitas } \\
\text { obstáculos a los } \\
\text { integrantes de tu }\end{array}$ & 13.45763932 & $\begin{array}{l}\text { La fuerza del } \\
\text { deseo por lograr } \\
\text { las metas genera } \\
\text { liderazgo al } \\
\text { quitar } \\
\text { obstáculos a los } \\
\end{array}$ \\
\hline
\end{tabular}




\begin{tabular}{|c|c|c|c|c|c|}
\hline & & $\begin{array}{l}\text { para su } \\
\text { crecimiento? }\end{array}$ & $\begin{array}{l}\text { equipo } \\
\text { emprendedor para } \\
\text { su crecimiento }\end{array}$ & & $\begin{array}{l}\text { integrantes del } \\
\text { equipo. }\end{array}$ \\
\hline$(6,5)$ & $\begin{array}{l}\text { ¿Qué tan } \\
\text { frecuente } \\
\text { mantienes tu } \\
\text { visión de las } \\
\text { metas a lograr? }\end{array}$ & $\begin{array}{l}\text { ¿Qué tan fuerte } \\
\text { es tu deseo por } \\
\text { lograr tus } \\
\text { metas? }\end{array}$ & $\begin{array}{l}\text { Si mantienes tu } \\
\text { visión de las metas } \\
\text { a lograr entonces } \\
\text { tienes un fuerte } \\
\text { deseo por llegar a } \\
\text { cumplirlas }\end{array}$ & 52.750427 & $\begin{array}{l}\text { El } \\
\text { mantenimiento } \\
\text { de una visión de } \\
\text { las metas a } \\
\text { lograr genera un } \\
\text { deseo ardiente } \\
\text { por cumplirlas }\end{array}$ \\
\hline$(7,9)$ & $\begin{array}{l}\text { ¿Qué tanta fe } \\
\text { tienes para } \\
\text { invertir o } \\
\text { conseguir } \\
\text { inversión para } \\
\text { lograr tu meta } \\
\text { emprendedora? }\end{array}$ & $\begin{array}{l}\text { ¿Con qué } \\
\text { frecuencia te } \\
\text { enfocas en tu } \\
\text { meta } \\
\text { emprendedora? }\end{array}$ & $\begin{array}{l}\text { Si tienes fe para } \\
\text { invertir o conseguir } \\
\text { inversión para } \\
\text { lograr tu meta } \\
\text { emprendedora, } \\
\text { entonces te enfocas } \\
\text { en tu meta } \\
\text { emprendedora. }\end{array}$ & 29.21208371 & $\begin{array}{l}\text { La fe para } \\
\text { invertir o } \\
\text { conseguir } \\
\text { inversión genera } \\
\text { un enfoque } \\
\text { permanente en } \\
\text { la meta } \\
\text { emprendedora }\end{array}$ \\
\hline$(12,10)$ & $\begin{array}{l}\text { ¿Qué tanto } \\
\text { aceptas y } \\
\text { superas los } \\
\text { reveses que se } \\
\text { presentan en el } \\
\text { camino a tu } \\
\text { meta? }\end{array}$ & $\begin{array}{l}\text { Cuándo tienes } \\
\text { un cambio } \\
\text { inesperado en el } \\
\text { camino hacia tu } \\
\text { meta } \\
\text { emprendedora } \\
\text { ¿Qué tan rápido } \\
\text { te adaptas a las } \\
\text { nuevas normas } \\
\text { o } \\
\text { características? }\end{array}$ & $\begin{array}{l}\text { Si aceptas y superas } \\
\text { reveses que se } \\
\text { presentan en el } \\
\text { camino a tu meta, } \\
\text { entonces te adaptas } \\
\text { a las nuevas norma } \\
\text { y características de } \\
\text { un cambio } \\
\text { inesperado }\end{array}$ & 53.66276934 & $\begin{array}{l}\text { Aceptar y } \\
\text { superar los } \\
\text { reveses que se } \\
\text { presentan en el } \\
\text { camino a la } \\
\text { meta genera } \\
\text { adaptación a las } \\
\text { nuevas normas y } \\
\text { características } \\
\text { de un cambio } \\
\text { inesperado }\end{array}$ \\
\hline
\end{tabular}

La tabla 4 tiene la misma descripción que la tabla 3, la diferencia se tiene en la columna 5. Esta columna enuncia el valor calculado u observada del chi cuadrado. Este valor se interpreta de la siguiente manera:

Hipótesis nula $\mathrm{H}_{\mathrm{o}}=$ las variables no están asociadas.

Hipótesis alternativa $\mathrm{H}_{1}=$ las variables están asociadas.

El valor de referencia del chi cuadrado para un contraste dicotómico con un grado de libertad y una significancia de 0.5 es de 3.84. Este valor se dice que es de chi cuadrado teórico o crítico.

Inferencias conclusivas:

1. Si el valor del chi cuadrado observado excede al 3.84, entonces se dice que la hipótesis nula se rechaza y se acepta la hipótesis alternativa. Por lo tanto, las variables están asociadas.

2. Si el valor de chi cuadrado observado es menor al 3.84, entonces se dice que la hipótesis nula se acepta y se concluye que las variables no están asociadas. 
En la tabla 4, se exhiben ocho hipótesis con valores arriba del 3.84. En la columna 5 se muestra cada una de las hipótesis comprobadas por medio del chi cuadrado.

Estas hipótesis de trabajo (de las 400 formuladas en una ordenación de los 20 ítems tomados de 2 en 2), fueron seleccionadas por los expertos debido al poco espacio de un artículo científico.

En relación con la discusión de los resultados vemos que, como dice Espíritu y Moreno (2011) que los estudiantes tienen una estrecha relación de la necesidad de logro, control interno y propensión al riesgo como factores de personalidad que potencian su intención de crear su propia empresa. Se observa claramente en las hipótesis comprobadas en esta investigación.

Por otro lado, estamos de acuerdo con Feliz J. (2017) que nos indica que los factores sociodemográficos no son determinantes en el desarrollo de una actitud emprendedora en los estudiantes, en cambio, factores psicológicos y sociológicos como el capital social, las redes sociales y el entorno familiar influyen de manera preponderante en su comportamiento emprendedor.

\section{Conclusiones}

Se hizo una investigación a cerca de la actitud emprendedora de los estudiantes varones del TecNM campus Tepeaca.

Se diseñó la matriz de operacionalización para tener una congruencia en la lógica menor y lógica mayor de la investigación.

Se sometió a una validación exhaustiva por expertos, del cuestionario que se aplicó a 172 sujetos de estudio.

El cuestionario se obtuvo de la matriz de operacionalización por medio de la técnica de Brian Tracy (lluvia de ideas).

Directamente se hizo un análisis multifactorial a través de la correlación múltiple de Pearson. A estos resultados se le aplicaron cinco postulados para su validación por expertos (nuevo método de validación).

Se hizo la misma técnica de validación con chi cuadrado para las asociaciones no lineales.

Las hipótesis de investigación se subdividieron en hipótesis de trabajo, tomando cada ítem como una variable independiente o factor.

Esta subdivisión se llevó a cabo debido a la facilidad y abundancia de hipótesis de trabajo para contrastar y validar. Técnica totalmente nueva.

Se validaron seis hipótesis lineales con la correlación de Pearson y más de treinta hipótesis no lineales con chi cuadrado. Solo se enuncian ocho de hipótesis no lineales por razones de espacio.

La metodología es totalmente nueva, no se comparó con otras metodologías para dejar que el público especializado la apruebe o desapruebe.

Como conclusión general. Los estudiantes varones del tecnológico nacional de México campus Tepeaca tienen un conocimiento muy alto en 
cuanto al emprendimiento, una excelente disposición para innovar y crear empresas de éxito. Por su abundante comprobación por chi cuadrado en la asociación de variables como hipótesis de trabajo, se concluye que sus pensamientos como sujetos de estudio es no lineal. Por otro lado, el lenguaje escogido para dar a conocer las hipótesis comprobadas es totalmente sencillo con el fin de que sea fácil de entender entre nuestros estudiantes.

\section{References:}

1. Borrayo, C., Zepeda, A. V., \& Melgarejo, B. D. (2019). Cultura emprendedora en jóvenes universitarios de Guadalajara, México. Revista de Ciencias Sociales, 25(3), 72-87.

2. Cruz, N. M., Barahona, J. J. H., \& Escudero, A. I. R. (2005). Análisis de la formación y la experiencia laboral como determinantes del espíritu emprendedor de los estudiantes universitarios. RAE: Revista Asturiana de Economía, (34), 131-145.

3. De Jorge, J. (2013). Análisis de los factores que influyen en la intención emprendedora de los estudiantes universitarios. Revista digital de investigación en docencia, 1 (1), 1-12

4. Espíritu, R., Moreno, H. (2011). La influencia de los rasgos de personalidad riesgo, control interno y necesidad de logro en la intención emprendedora: un estudio empírico con estudiantes universitarios. XV Congreso Internacional de Contaduría, Administración e Informática. ANFECA, 1-33.

5. Feliz, J. (2017). Capital social, redes sociales y actitud emprendedora en estudiantes universitarios de Rep. Dom. Ciencia y Sociedad, 42(1),43-58.

6. García-Arroyo, L.C., Gómez-Márquez, M. \& Méndez-Exzacariast, V. (2020a). Institutionalization of the family business through the creation of a protocol to achieve the continuity. Global Journal of Engineering Science and Research Management, 7(9).

7. García-Arroyo, L.C., Gómez-Márquez, M. \& Quintero-Fuentes, M. (2020b). The Successor Training as a Success Factor in the Management and Continuity of the Family Business. European Scientific Journal, ESJ, 16(31).

8. Hernández, R. (2019). Intención emprendedora: ¿es influenciada por factores de personalidad y género? Revista Academia \& Negocios, 4 (2), 1-12.

9. Méndez, E., Aguirre, J., \& Flores, M. (2017). Análisis Comparativo De La Intención Emprendedora En Alumnos De Diversos Semestres y Carreras De La Facultad De Contaduría y Administración De La Universidad Autónoma De Chihuahua. Las ciencias informáticas, $\left(1^{\mathrm{a}}\right.$ ED) 121-137. 
10. Neira, I., Portela, M., Fernández, L., \& Rodeiro, D. (2013). Actitud emprendedora: un enfoque a través de los jóvenes y el género. Una perspectiva de género, (5), 69-84.

11. Olmos, E. (2011). Análisis de la intención emprendedora en estudiantes universitarios a través de los rasgos de personalidad. Multiciencias, 11(1), 65-75.

12. Ordóñez, C. A. G. (2017). Habilidades emprendedoras en los estudiantes de la carrera de Gestión Empresarial de la Universidad Metropolitana, Sede Machala. Revista Conrado, 13(1), 122-131.

13. Rincón, D., \& Álvarez, D. (2020). Espíritu emprendedor universitario, desde una perspectiva de género, estudio empírico del estado de Guanajuato, México. Tendencias en la Investigación Universitaria: Una visión desde Latinoamérica, volumen X, 117-131.

14. Ruiz, C. I., García, F., \& Delgado, N. (2014). Condicionantes de la intención emprendedora en el alumnado universitario: un análisis desde la perspectiva de género. Revista de Estudios Empresariales. Segunda Época, (2), 81-96. 